\title{
Anti-submarine Helicopters' Cooperative Submarine Detecting Fan Sector Modeling
}

\author{
Li Xinshu ${ }^{\mathrm{a}}$, Mao Zhongyang ${ }^{\mathrm{b}}$, Zhang Yang $^{\mathrm{c}}$, Li Weibo ${ }^{\mathrm{d}}$
}

Naval Aeronautical Engineering Institute Yantai, Shandong, China

axinssh@sina.com, b kinleb227@126.com, cZY83051@sina.com, dsunlight2637@163.com

Key words: Submarine detecting fan sector; Dynamic calculation; Dipping sonar; Anti-submarine helicopter; Detection area

\section{Abstract.}

This paper is aiming at the submarine detecting fan sector dynamic calculation problem of anti-submarine helicopters using dipping sonar search. Helicopters hovering point coordinate solution model is established based on search method. The equivalent method is adopted to calculate submarine alert area when the current hovering point dipping sonar's detection area is equivalent to the area where all the hovering points finished detection do not exist. Every hovering point detective area's dynamic detection model relative to naval warship formation angle is established, and dynamic calculation methods of the beginning and ending of helicopters cooperative submarine detecting fan sector and ending angle are given. Finally, the dynamic relationship between submarine detecting fan sector and search time, spacing coefficient is calculated. Results show that submarine detecting fan sector changes periodically in certain range and is more influenced by spacing coefficient.

\section{Introduction}

Anti-submarine helicopters implement escort anti-submarine around a certain distance of surface warship formation (such as aircraft carrier formation), which is an important way against the threat of underwater submarines. Dipping sonar [1-2] is a unique anti-submarine equipment of helicopter search, which has merits like reusable, large distance effect and high search efficiency; by helicopter maneuver, it can get the best location to detect and can better meet the need of surface warship for submarine combats.

Submarine detecting fan sector is an important indicator of anti-submarine helicopter to accompany escort anti-submarine using dipping sonar [3-4]. As anti-submarine helicopters use dipping sonar search, they have to make repetitive hovering detection and maneuver flight between hovering points, resulting in a dynamic change of submarine detecting fan sector. Coupled with that warship formation is in sailing, this dynamic condition is more complex. At present, there are a lot domestic and foreign research on anti-submarine helicopters using dipping sonar search method, but the domestic dipping sonar submarine detecting fan sector research mainly adopts static analysis method to calculate, and the impact of submarine maneuver on alert fan sector is not taken into account.

\section{Anti-submarine helicopter dipping sonar submarine detecting fan sector}

In order to defend against underwater submarine threat, surface warship formation usually dispatch a number of carrier-based anti-submarine helicopters to carry out escort submarine detection on the carriers or around the formation, which is shown in Figure 1. 


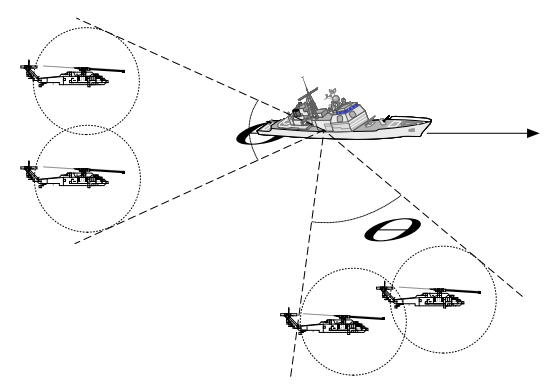

Figure 1 Anti-submarine helicopter dipping sonar submarine detecting fan sector diagram

Submarine detecting fan sector, angle $\theta$ as shown in Figure 1, refers to anti-submarine helicopter detection area which can cover the scope of warship angle. It is one of the key parameters in combat decision-making. Obviously, the bigger submarine detecting fan sector, the better; but in fact, due to the limited number of ship-borne anti-submarine helicopters, we can only implement effective submarine detection alert in a certain fan sector.

\section{Dynamic calculation method of helicopters dipping sonar submarine detecting fan sector}

As anti-submarine helicopters can search in directions such as front, flank and tail of the surface warship formation, calculation of submarine detecting fan sector includes the above-mentioned directions, and the calculation method and steps are the same. Take anti-submarine helicopters searching behind surface warships tail as an example. The dynamic calculation method of submarine detecting fan sector is studied.

Warship formation direction is X-axis, anti-submarine helicopter's first hovering point, the vertical $\mathrm{X}$-axis direction is Y-axis, and two-axis intersection is the origin coordinates. Establish rectangular coordinate system, which is shown in Figure 2.

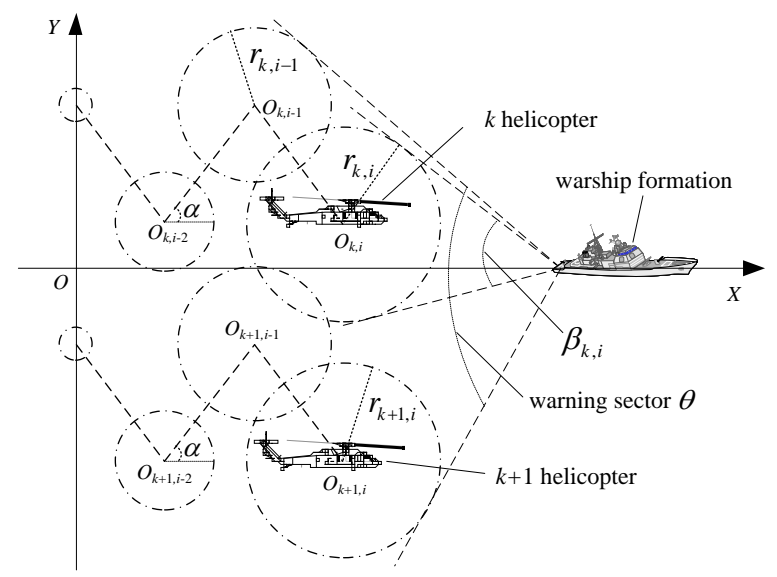

Figure 2 Dynamic calculation of dipping sonar search submarine fan sector diagram

Dynamic calculation of helicopters dipping sonar search submarine fan sector mainly includes the following four steps:

Step 1: Solve the coordinates of all hovering points for each anti-submarine helicopter;

At the time of the first use of dipping sonar, the moment is $0, D_{0}$ is the X-coordinate of warship formation center, the first hovering point coordinate $O_{k, 1}\left(x_{k, 1}, y_{k, 1}\right)$ of $k$ helicopter is

$$
\left\{\begin{array}{l}
x_{k, 1}=0 \\
y_{k, 1}=y_{k-1,1}-(k-1) d_{\text {hel, jg }} \quad k>1
\end{array}\right.
$$

In it, $d_{\text {hel,jg }}$ is the distance between adjacent anti-submarine helicopters.

Anti-submarine helicopters mainly use serration method in the search behind warships formation [17]. In order to make the search area evenly distributed on both sides of formation route, the coordinate $O_{1,1}\left(x_{1,1}, y_{1,1}\right)$ of the first hovering point for the first helicopter shall meet 


$$
\left\{\begin{array}{l}
x_{1,1}=0 \\
y_{1,1}=\frac{1}{2} \cdot d_{\mathrm{jg}} \cdot \sin (\alpha)+\frac{N_{\mathrm{hel}}-1}{2} \cdot d_{\mathrm{hel}, \mathrm{gg}}
\end{array}\right.
$$

In them, $N_{\text {hel }}$ is the number of dispatched anti-submarine helicopters; $d_{\text {jg }}$ isthe distance between adjacent hovering points, $\alpha$ is the angle of distance between the adjacent hovering points and warship formation direction.

According to the characteristics of dogleg method submarine detection, coordinate $O_{k, i}\left(x_{k, i}, y_{k, i}\right)$ of the $(i>1)$ hovering point of $k$ helicopter is

$$
\left\{\begin{array}{l}
x_{k, i}=x_{k, i-1}+d_{\mathrm{jg}} \cdot \cos (\alpha) \\
y_{k, i}=y_{k, i-1}+(-1)^{i-1} \cdot d_{\mathrm{jg}} \cdot \sin (\alpha)
\end{array}\right.
$$

In them, $d_{\mathrm{jg}}$ is the product of dipping sonar effective function $R_{\text {dipson }}$ distance and the spacing factor $\xi_{\text {dipson }}$, which is

$$
d_{\text {jg }}=R_{\text {dipson }} \xi_{\text {dipson }}
$$

In order to make the hovering point distribution symmetrical, take

$$
d_{\text {hel, }, \mathrm{jg}}=2 d_{j g} \sin (\alpha)
$$

Step 2: Use equivalent method to solve the area of dipping sonar for submarine search;

At present, the calculation methods of dipping sonar search area only consider the detection range of the current hovering point, and ignore the sea area where the search has been completed. In fact, dipping sonar search area includes not only the current detection area but also the detected areas where submarines do not exist in the water.

The sea range of no submarine after complete search is reduced to 0 with any maneuver of the submarine gradually. Therefore, equivalent method is used to equalize the sea area of no submarine around the hovering point that has been searched completely in several circles and all equalized circle areas are dipping sonar search areas.

As the submarine target is in constant motion, it means that $i$ hovering point which is an area without target, will continue to shrink over time to 0 after the end of the hearing, then

$$
r_{k, i}(t)=\left\{\begin{array}{lc}
0 & t<t_{k, i, s \text { tart }} \\
R_{\text {dipson }} & t \in\left[t_{k, i, \text { start }}, t_{k, i, \text { end }}\right] \\
\max \left\{0, R_{\text {dipson }}-\bar{v}_{\text {sub }}\left(t-t_{k, i, \text { end }}\right)\right\} & t>t_{k, i, \text { end }}
\end{array}\right.
$$

In it, $r_{k, i}(t)$ is the equivalent radius of the target area without submarine at $r_{k, i}(t)$ hovering point of $k$ helicopter; $\bar{v}_{\text {sub }}$ is the mean value of submarine's target speed; $t_{k, i, \text { start }}$ and $t_{k, i, \text { end }}$ respectively, indicate $k$ helicopter's beginning and ending hearing time at $i$ hovering point.

According to the search process of anti-submarine helicopter using dipping sonar, the actual duration of underwater detection at each hovering point is only $T_{\text {dipson,listen }}$, dipping sonar underwater transducers' hearing time, then

$$
\left\{\begin{array}{l}
t_{k, i, \text { start }}=(i-1) \cdot T_{\text {dipson,cyc }} \\
t_{k, i, \text { end }}=(i-1) \cdot T_{\text {dipson,cyc }}+T_{\text {dipson,listen }}
\end{array}\right.
$$

In it, $T_{\text {dsonar,cyc }}$ is the search cycle of anti-submarine helicopters using dipping sonar.

Step 3: Dynamically calculate the angle of each hovering point detection area relative to warship formation;

Make $\psi_{k, i, \text { cent }}$ warship formation center angle relative to hovering point $O_{k, i}, \beta_{k, i}$ is submarine detecting fan sector's center angle formed by $O_{k, i}$ point dipping sonar detection area, there are 
$\begin{cases}\psi_{k, i, c \text { ent }}(t)= \begin{cases}\pi-\arcsin \left(\frac{\Delta y_{k, i}(t)}{d_{k, i}(t)}\right) & \Delta y_{k, i} \geq 0 \\ -\pi-\arcsin \left(\frac{\Delta y_{k, i}(t)}{d_{k, i}(t)}\right) & \Delta y_{k, i}<0\end{cases} \\ \beta_{k, i}(t)=2 \cdot \arcsin \left(\frac{r_{k, i}(t)}{d_{k, i}(t)}\right)\end{cases}$

Where $\Delta y_{k, i}(t)=y_{k, i}-y_{\text {ship }}(t), \quad d_{k, i}(t)=\sqrt{\left(x_{k, i}(t)-x_{\text {ship }}(t)\right)^{2}+\left(\Delta y_{i}(t)\right)^{2}} ; d_{k, i}$ is the distance between hovering point $O_{k, i}$ and warship formation center; $\left(x_{\text {ship }}, y_{\text {ship }}\right)$ isthe coordinates of the center position of the warship formation, make $V_{\text {ship }}$ the speed of warship formation, then

$$
\left\{\begin{array}{l}
x_{\text {ship }}(t)=D_{0}+V_{\text {ship }} \cdot t \\
y_{\text {ship }}(t)=0
\end{array}\right.
$$

In order to facilitate the calculation, it is desirable to take $\psi_{k, i, \mathrm{cent}}(t)=\pi-\arcsin \left(\frac{\Delta y_{k, i}(t)}{d_{k, i}(t)}\right)$; if $\psi_{k, i, \text { cent }}>\pi, O_{k, i}$ is located on the starboard side, take $\psi_{k, i, \text { cent }}(t)-2 \pi$, which is the actual port angle.

Step 4: Dynamic calculation of submarine detecting fan sector range covered by helicopters dipping sonar search area.

The beginning angle $\psi_{k, i, \text { start }}(t)$ and the ending angle $\psi_{k, i, \text { end }}(t)$ of $k$ helicopter's $i$ hovering point detection area covered submarine detecting fan sector are respectively,

$$
\left\{\begin{array}{l}
\psi_{k, i, \text { start }}(t)=\psi_{k, i, \text { cent }}(t)-\beta_{k, i}(t) / 2 \\
\psi_{k, i, \text { end }}(t)=\psi_{k, i, \text { eent }}(t)+\beta_{k, i}(t) / 2
\end{array}\right.
$$

According to Equations (10), the beginning angle and the ending angle of each anti-submarine helicopter and each hovering point without cover of target area at any time $t$ can be calculated; if there is overlap between the sectors, merge into one fan; select the largest central angle fan from the center as the submarine detecting fan sector covered by anti-submarine helicopter search area.

\section{Simulation analysis}

The surface warship formation is navigating at 20 knots, and the X-coordinate $D_{0}$ at the center of warships formation is 15 nautical miles at time 0 . The number of helicopters dispatched is $N_{\text {hel }}=3$, dipping sonar search cycle $T_{\text {dsonar,cyc }}$ is 12 minutes, the hearing time $T_{\text {dipson,listen }}$ is 6 minutes at each detection point, 20-speed submarines' effective detection range is $10 \mathrm{~km}$; to achieve the continuous tracking of surface warship formation, the average speed is of the same as formation sailing speed.

Respectively take spacing coefficient of 1.01 and 1.63 for simulation, and the results are shown in Figure 3.

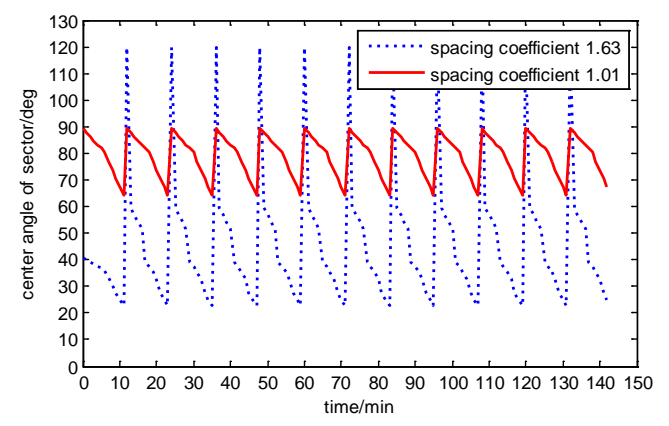

Figure 3 Submarine detecting fan sector dynamic changes with search time

Figure 3 shows the process of dynamic search changes of submarine detecting fan sector with 
time. It can be seen from the Figure that searching during the first two hovering points, due to the small number of detected zones, there has not formed stable sector. The sector will periodically change over a range of angles, and the change period is the search cycle of dipping sonar. When the spacing factor is 1.63 , submarine detecting fan sector can obtain a relatively short $119.8^{\circ}$ segment, but then it rapidly decreases to a minimum of $22.57^{\circ}$, and the variation of fan center angle is large. While the spacing factor is 1.01, although the submarine detecting fan sector is not greater than $89.61^{\circ}$, it is not less than $64.36^{\circ}$, and the variation range is narrow.

For other spacing coefficients, the range of submarine detecting fan sector dynamic changes can also be obtained. The simulation results are shown in Figure 4.

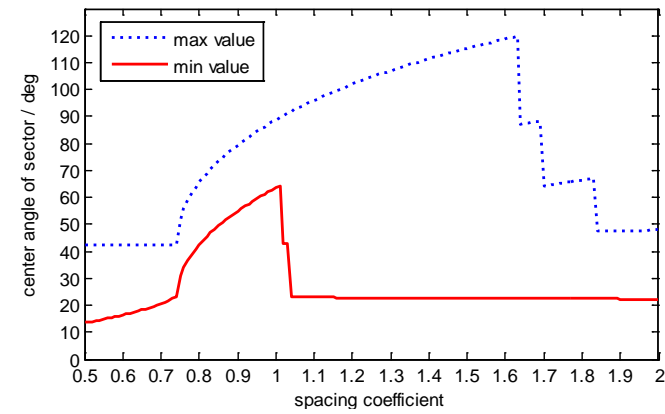

Figure 4 Dynamic changes of submarine detecting fan sector with the change of spacing coefficient

In the Figure, blue dotted line is the maximum value of the submarine detecting fan sector under different spacing coefficient, and the red solid line is the minimum value. That is, fan center angle will change dynamically between the red solid line and the blue dotted line in the Figure. In order to ensure the tightness of submarine defense, it should make the minimum value of submarine detecting fan sector as large as possible, and we can take the maximum figure in the red line, which is $64.36^{\circ}$. This means that in entire search process, submarine detecting fan sector will not be less than $64.36^{\circ}$, and the corresponding spacing coefficient is 1.01 ; at this time the change process of submarine detecting fan sector with time is shown as the red solid line in Figure 3.

\section{Conclusion}

Anti-submarine helicopters use dipping sonar "jump" type search and accompany escort anti-submarine surface warships' rapid navigation, which makes the dynamic character of submarine detecting fan sector particularly obvious. Through modeling and simulation, the following is obtained:

(1) Submarine detecting fan sector will have cyclical dynamic changes in a certain range, and the cycle is dipping sonar's search cycle;

(2) Spacing coefficient affects submarine detecting fan sector. When taking 1.01, submarine detecting fan sector is no less than $64.36^{\circ}$; if take 1.63 , the maximum of submarine detecting fan sector is $119.8^{\circ}$, but then it quickly drops to a minimum of $22.57^{\circ}$.

To sum up, this article provides a dynamic calculation of helicopters dipping sonar submarine detecting fan sector approach. Taking into account the current hovering point dipping sonar detection area and all completed detection hovering points areas without submarine, the dynamic calculation result of submarine detecting fan sector becomes more realistic, which can provide more accurate decision reference for anti-submarine warfare decision.

\section{References}

[1] Qi Xuewen, Yan Jiangang, Xie Yu. Helicopter Dipping Sonar Recall Submarine Detecting Model [J]. Fire and Command \& Control, 2014, 39 (3): 47-49.

[2] Li Xiangke, Zhao Zhiyun, Du Yi. Anti-submarine helicopter dipping sonar passive search potential simulation and optimization [J]. Electro-optical and control, 2015,22 (11): 95-99. 
[3] Cong Hongri, Shen Peizhi, Wang Wei. Helicopter flanking method with escort anti-submarine warfare method and its simulation [J]. Ship Science and Technology, 2011,33 (3): 115-120.

[4] Cong Hongri, Xiao Mingqiang, Chen Dengan. Synchronization Research on Helicopter Anti-submarine Patrol Line and Formation [J]. School of Electronics Engineering, 2011,31 (8): 8-10.

[5] Wu Ming. Dipping fan sector helix search diving modeling based on auspicious target heading [J]. Journal of System Simulation, 2015,27 (12): 2908-2912. 